\title{
EGFR Expression Is Unreliable to Decide for Cetuximab Treatment in Patients with Metastatic Colorectal Cancer: Results from "ERBITUX-OUEST"
}

Jean-Philippe Metges, ${ }^{1,2}$, Erick Gamelin', Alain Volant'2, Olivier Capitain ${ }^{3}$, Jean-François Ramée ${ }^{4}$, Jean-Luc Raoul ${ }^{5}$, Jean-Yves Douillard ${ }^{1,6}$, Pierre-Luc Etienne ${ }^{7}$, Isabelle Cumin' ${ }^{8}$, Olivier Dupuis ${ }^{9}$, Roger Faroux ${ }^{10}$, Marie-Aude Coulon ${ }^{11,23}$, Philippe Deguiral12, Karine Bideau'3, Nacr Eddine Achour14, Alain Gourlaouen15, Corinne Alleaume ${ }^{16}$, Annie Wdowik ${ }^{17}$, Laurent Miglianico ${ }^{18,26}$, Yann Touchefeu ${ }^{19}$, Vincent Klein ${ }^{20}$, Alain Penchet ${ }^{21,24}$, Ludovic Rosenfeld ${ }^{22}$, Daniel Martin ${ }^{23}$, Claire Stampfli25, Jean-Jacques Auger ${ }^{27}$, Alain Bargain ${ }^{28}$, Murielle Broyer-Petit ${ }^{29}$, Jérome Chettrit ${ }^{30}$, Louis-Rémi De Ybarlucea11, Serge Eloit ${ }^{31}$, Pierre Marie Girardot ${ }^{32}$, Nathalie Heresbach ${ }^{33}$, Christian Laboisse ${ }^{19}$, Eric Lavoine ${ }^{34}$, Gilles Lemasson ${ }^{35}$, Claire Magois ${ }^{10}$, Sophie Michalak ${ }^{36}$, Norbert Padilla ${ }^{37}$, Joseph Politis ${ }^{38}$, Frédéric Staroz ${ }^{39}$, Bruno Turlin ${ }^{40}$, Arnaud Uguen², Viorel Vasiliu37, Véronique Verriele ${ }^{3}$, Fanny Marhuenda1, Delphine Déniel Lagadec ${ }^{1}$, Christian Riché ${ }^{1}$, Françoise Grudé ${ }^{1}$

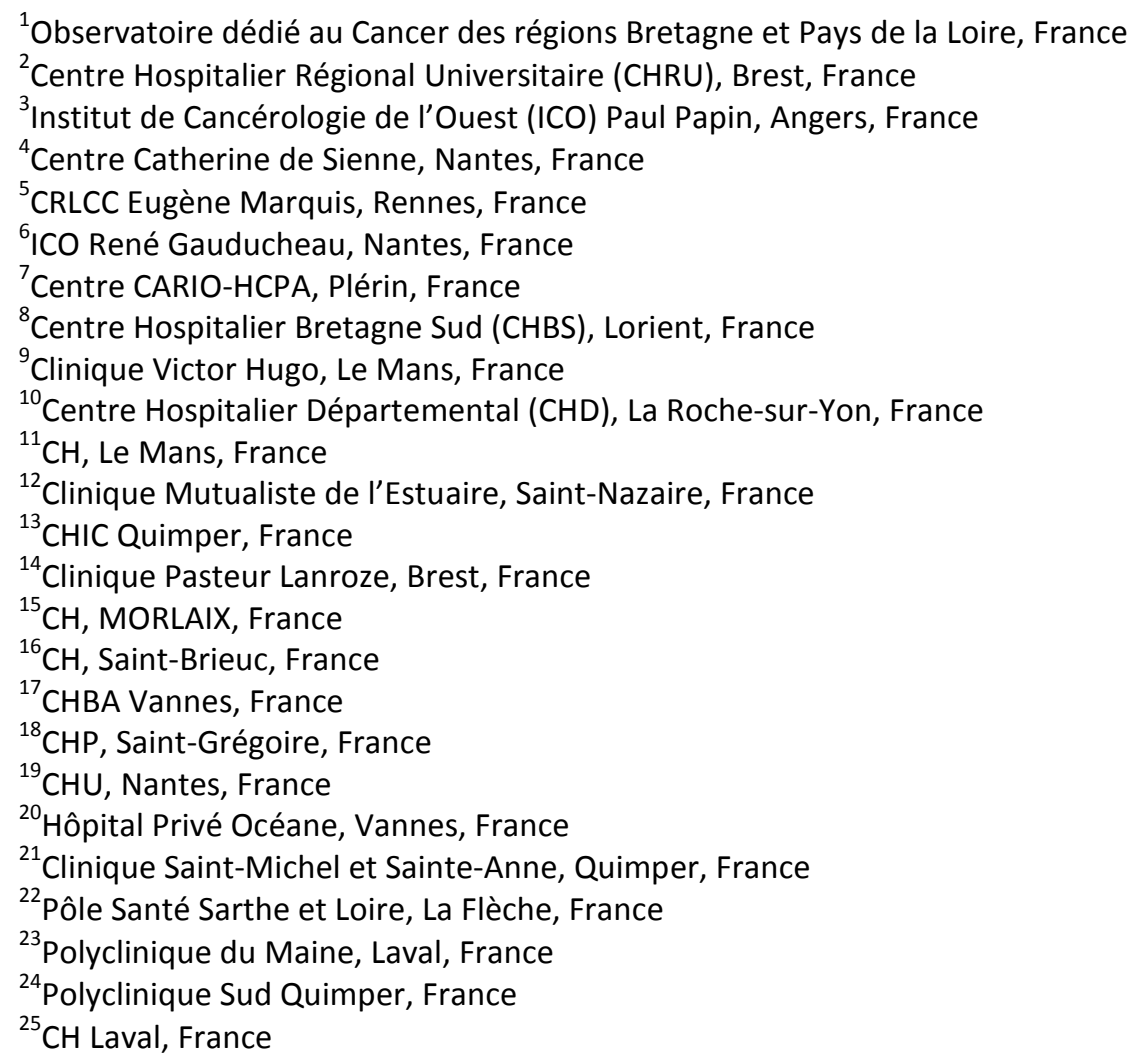

How to cite this paper: Metges, J.-P., et al. (2016) EGFR Expression Is Unreliable to Decide for Cetuximab Treatment in Patients with Metastatic Colorectal Cancer: Results from "ERBITUX-OUEST". Open Journal of Internal Medicine, 6, 56-67. http://dx.doi.org/10.4236/ojim.2016.62010 
${ }^{26}$ Polyclinique Cesson Sévigné, France

${ }^{27}$ Laboratoire d'anatomie et de cytologie pathologiques, Cholet, France

${ }^{28}$ Cabinet d'anatomie et de cytologie pathologiques, Saint-Malo, France

${ }^{29}$ Laboratoire d'anatomie et de cytologie pathologiques, Lorient, France

${ }^{30}$ Institut d'histopathologie, Nantes, France

${ }^{31}$ Laboratoire de Pathologie Médicale, Laval, France

${ }^{32}$ ARMOR Pathologie, Plérin, France

${ }^{33}$ Laboratoire d'anatomie et de cytologie pathologiques, Rennes, France

${ }^{34}$ Laboratoire d'anatomie et de cytologie pathologiques, Angers, France

${ }^{35}$ Laboratoire Analyses Biologie Glasgow, Brest, France

${ }^{36} \mathrm{CHU}$ Angers, France

${ }^{37}$ Centre d'anatomie et de cytologie pathologiques, Le Mans, France

${ }^{38}$ Cabinet d'anatomie et de cytologie pathologiques, Vannes, France

${ }^{39}$ Laboratoire d'anatomie et de cytologie pathologiques, Quimper, France

${ }^{40} \mathrm{CHU}$ Rennes, France

Email: "jean-philippe.metges@chu-brest.fr

Received 30 March 2016; accepted 19 June 2016; published 22 June 2016

Copyright (C) 2016 by authors and Scientific Research Publishing Inc.

This work is licensed under the Creative Commons Attribution International License (CC BY). http://creativecommons.org/licenses/by/4.0/

(c) (i) Open Access

\section{Abstract}

Purpose: Although controversial, assessment of epidermal growth factor receptor (EGFR) expression is required for the approved indications of Cetuximab in metastatic colorectal cancer (mCRC). With the objective of improving patient selection, "ERBITUX-OUEST" study aimed at analyzing EGFR status in a large cohort of mCRC patients who received cetuximab without preliminary EGFR screening, and assessing the correlation between EGFR status and response to treatment retrospectively. Patients and methods: 332 patients treated with Irinotecan Cetuximab based regimen after progression on irinotecan or oxaliplatin therapy were included. EGFR status was assessed using three available immunohistochemistry (IHC) tests and in situ hybridization in case of negativity. Clinical outcomes of EGFR-positive and EGFR-non-detected (or considered as negative with at least one test) patients were compared. Results: Of the 332 samples centrally screened, 194 were classified as full-positive (i.e., EGFR-positive for all three tests), 86 as full-negative, and 52 as discordant. One third of the 131 negative samples with FDA approved test should be reclassified as positive with at least one of the two others tests. Regarding results from FDA approved test only, neither objective response rate (ORR), progression-free survival (PFS) nor overall survival (OS) differed significantly between EGFR-negative and EGFR-positive patients $(P=0.788,0.326$ and 0.888, respectively). Similarly, comparison of full-negative to other groups did not show any significant difference in terms of ORR $(P=0.507)$, PFS $(P=0.222)$ or OS $(P=0.686)$. Conclusion: These data strongly argue against mCRC patients selection for Cetuximab treatment based on EGFR expression as measured by currently available IHC technics.

\section{Keywords}

Metastatic Colorectal Cancer, Cetuximab, EGFR Expression

${ }^{*}$ Corresponding author. 


\section{Introduction}

Colorectal cancer (CRC) is a very common disease with a high rate of mortality around the world representing the second cause of cancer-related death [1]. Based on the theoretical assumption that the EGFR status of a tumor may provide an indication of the likelihood of response to EGFR-targeted therapy, studies that demonstrated activity of cetuximab in mCRC have been restricted to those patients whose tumors expressed EGFR by immunohistochemistry (IHC). Thus, assessment of IHC expression of EGFR has been mandatory before initiation of cetuximab based therapy. In this context, a standardized IHC kit (Dako's EGFR pharmDx ${ }^{\mathrm{TM}}$ kit) was developed and approved by the Food and Drug Administration (FDA) in 2004 as an aid in identifying CRC patients eligible for treatment with cetuximab. Currently, regulatory agencies still require selection of patients for cetuximab-based therapy by IHC and several kits have been developed that can indifferently be used for EGFR status assessment.

However, the linear correlation between the degree of EGFR expression, as detected by IHC and response to cetuximab is being questioned. On the one hand, clinical studies have suggested that EGFR staining intensity does not correlate with clinical outcomes in patients treated with cetuximab [2]-[7]. On the other hand, some studies showed that even patients with tumors non-detected for EGFR might benefit from this drug [6] [8]-[11]. Moreover, patients with negative immunostaining could have response: Wierzbicki et al. described $8.2 \%$ of response rate [12] in patients with progressive disease on previous treatment. The median duration of response was 5.1 months and $42.7 \%$ of patients had a stable disease.

All together, these observations strongly challenge the usefulness of IHC as a screen to select patients for cetuximab treatment. Possible causes for this paradox include false negative results due to a lack of sensitivity in the detection system, heterogeneity of EGFR expression within the tumor and underestimation of biologically active receptors [13]. Of note, however, these studies were carried out on small data sets of patients and their results have to be confirmed on larger cohorts.

Thus, to clarify the issue regarding the best criteria for selecting CRC patients who will most likely benefit from EGFR-targeted therapy, Bretagne-Pays de Loire areas (B PL) Cancer Observatory in France has decided to allow initiation of cetuximab-based treatment in CRC patients without mandatory preliminary EGFR status assessment. The BPL Cancer Observatory, an oncology network created by the French Ministry of Health, is specifically dedicated to assessing the use of new targeted anticancer therapies in routine practice. By gathering data from all patients treated in the 47 public hospitals and private institutions members of the network, a major role of B PL Cancer Observatory is to conduct large registry-based observational studies in various fields of oncology to issue recommendations for good medical practice.

The aim of "ERBITUX-OUEST" study was to retrospectively analyze data from the Cancer Observatory cohort of patients with mCRC treated with cetuximab regardless of their EGFR status, allowing, for the first time, to correlate the EGFR status, assessed using the three currently available IHC tests, with outcome in a large reallife cohort.

\section{Patients and Methods}

\subsection{Patients}

In the "ERBITUX-OUEST" study, tumor samples and clinical data were retrospectively collected from mCRC patients who had been treated with cetuximab-based therapy for metastatic disease in a center of the B PL Cancer Observatory network from January 2005 to December 2006. Patients have been followed up until death (or lost of follow up). During this period, neither assessment of EGFR status nor that of Kirsten rat sarcoma viral oncogene homolog (KRAS) was required for treatment initiation. All patients received a cetuximab-based regimen $(250 \mathrm{mg} / \mathrm{msq}$ weekly) in combination with irinotecan $(180 \mathrm{mg} / \mathrm{msq}$ biweekly) after irinotecan or oxaliplatin failure. Disease assessments were performed according to usual clinical practices.

This cohort was declared to the Commission Nationale de l'Informatique et des Libertés (CNIL) as a retrospective study without impact on patient treatment. Patients included in the cohort have a right of access, modification and deletion of the processing of their personal data. Excemption of inform duty had been obtained for deceased patients.

\subsection{EGFR Expression Status}

Centralized assessment of EGFR expression was performed for all tumor samples collected using the three 
commercially available kits: the FDA-approved Dako EGFR pharmDx ${ }^{\mathrm{TM}}$ kit (Dako test), Zymed EGFR kit from Zymed $^{\circledR}$ Laboratories (Zymed test) and Ventana CONFIRM ${ }^{\text {TM }}$ EGFR 3 C6 antibody from Ventana ${ }^{\circledR}$ (Ventana test), according to the manufacturer's protocol.

The staining results were measured semi-quantitatively on a scale of $0,1+, 2+$ and $3+$, as previously described [14] [15]. Briefly, a stain was scored as follows: 0, there is no membranous staining in any of the tumor cells or staining in less than $10 \%$ of the tumor cells; $1+$, there is incomplete membranous staining in $>10 \%$ of the tumor cells with weak intensity; $2+$, there is complete membranous staining in $>10 \%$ of the tumor cells with weak to moderate intensity; and $3+$, there is complete membranous staining in $>10 \%$ of the tumor cells with moderate to strong intensity. Full-negative patients were defined as EGFR non-detected for all the three IHC kits. Discordant cases were defined as patients with positive EGFR status in one or two of the three IHC kits. Figure 1 illustrates typical examples of full-negative and discordant cases.

\subsection{EGFR Gene Amplification Pattern}

Centralized assessment of EGFR gene amplification was performed systematically for patients with negative Dako test and for full-negative patients by automated silver-enhanced in situ hybridization (SISH) technique using the INFORM EGFR and Chromosome 7 centromere $(\mathrm{Chr} 7)$ probes, according to the manufacturer's protocol. Both probes were labelled with dinitrophenol and optimally formulated for use with the ultraVIEW SISH Detection Kit and accessory reagents on the Benchmark ${ }^{\circledR}$ XT machine (Ventana). EGFR and Chr7 signals were counted in 20 cells in a suitable tumor target area. Then the ratio between EGFR and $C h r 7$ signals was calculated. A $E G F R / C h r 7$ ratio of less than 2 was defined as negative for EGFR gene amplification; a $E G F R / C h r 7$ ratio between 2.2 and 4 was defined as a weak amplification of the EGFR gene; and a $E G F R / C h r 7$ ratio more than 4 was defined as a strong amplification of the EGFR gene.

\subsection{Clinical Outcome}

Clinical records were reviewed to collect efficacy and safety data. Efficacy criteria include objective response, progression-free survival (PFS) and overall survival (OS).

\subsection{Statistical Analysis}

OS was defined as the interval between start of therapy to death or last follow-up visit. PFS was defined as the interval between start of therapy to clinical progression or death. The Kaplan-Meier method was used to calculate median durations of OS and PFS and to plot survival curves. Comparison of survival times was carried out using log-rank tests of significance. Comparisons of data according to EGFR status were performed using the Pearson's Chi-2 test. Two-sided $P$ values of less than 0.05 were considered statistically significant. All parameters were calculated with a 95\% confidence interval (CI). All analyses were performed using SPSS for Windows, version 11.0 (SPSS Inc, Chicago, IL).

\section{Results}

\subsection{Patient Characteristics}

The cohort consisted of 559 patients who were treated with cetuximab from January 2005 to December 2006. Tumor samples and full clinical data (efficacy and safety) were retrospectively collected for 332 of them, allowing their inclusion in "ERBITUX-OUEST" study. Main characteristics of these patients are listed in Table 1.

Most patients had metastatic disease at the time of primary diagnosis $(61 \%)$ and all patient at the time of cetuximab based treatment. The median number of previous treatment lines was 3 (1-7), with a median number of treatment cycles of 6 (1-45). Cetuximab was administered mainly in second and third-line treatment $(25 \%$ and $54 \%$, respectively) in combination with either irinotecan alone $(82 \%)$ or with irinotecan/5-fluorouracil/folinic acid (FOLFIRI) regimen (18\%) after oxaliplatin or irinotecan failure.

\subsection{EGFR Status Evaluated by IHC and SISH Analysis}

EGFR IHC was successfully performed on the 332 tumor samples (128 primary tumors and 204 metastases) from 332 patients. 


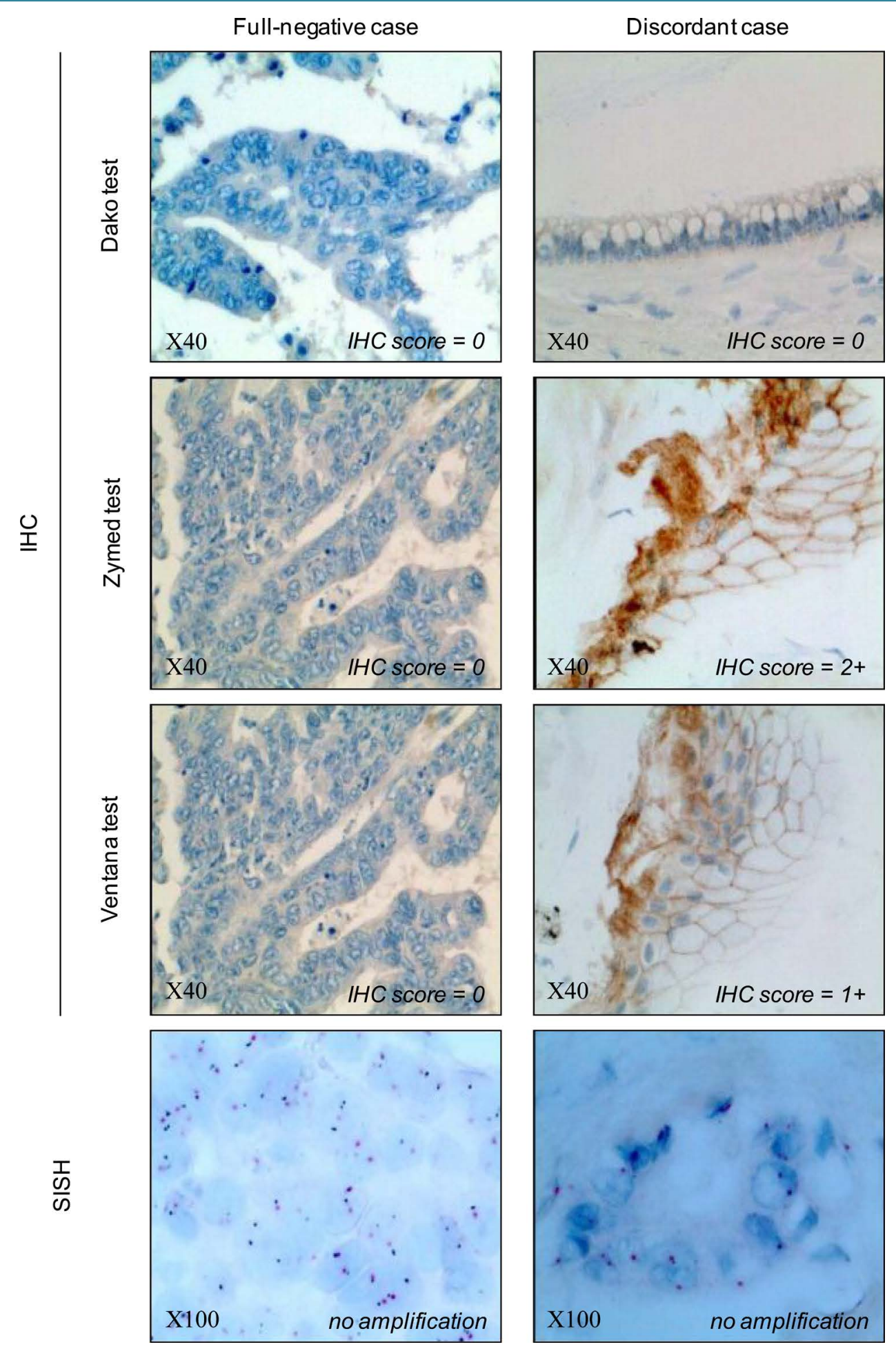

EGFR, epidermal growth factor receptor; IHC, immunohistochemistry; SISH, silver-enhanced in situ hybridization.

Figure 1. Representative EGFR staining pattern in tumor sample defined as full-negative case or discordant case by IHC. EGFR expression was assessed using the three commercially available kits: Dako's EGFR pharmDx ${ }^{\mathrm{TM}}$ kit (Dako test), Zymed EGFR kit from Zymed ${ }^{\circledR}$ Laboratories (Zymed test) and Ventana CONFIRMTM EGFR 3 C6 antibody from Ventana ${ }^{\circledR}$ (Ventana test). Corresponding IHC scores are indicated on each panel (magnification $\times 40$ ). The IHC results were interpreted using the scoring scheme proposed for gastric cancer by Hofmann [14] and Rüschoff [15]. Absence of EGFR expression was confirmed by absence of EGFR gene amplification as assessed by SISH (magnification $\times 100$ ). 
Table 1. Main patient characteristics.

\begin{tabular}{|c|c|c|}
\hline \multicolumn{2}{|c|}{ Characteristic } & $\mathbf{N}=332$ \\
\hline \multicolumn{2}{|c|}{ Median age (years, range) } & $64(33-83)$ \\
\hline \multirow{2}{*}{ Sex } & Male & $212(64 \%)$ \\
\hline & Female & $120(36 \%)$ \\
\hline \multirow{4}{*}{ ECOG status* } & 0 & $41(51 \%)$ \\
\hline & 1 & $33(41 \%)$ \\
\hline & 2 & $6(7 \%)$ \\
\hline & 3 & $1(1 \%)$ \\
\hline \multirow{4}{*}{ Tumor site $\dagger$} & Colon & $218(66 \%)$ \\
\hline & Rectum & $81(24 \%)$ \\
\hline & Recto-sigmoid junction & $29(9 \%)$ \\
\hline & Colon + rectum & $3(1 \%)$ \\
\hline \multirow{4}{*}{ Prior treatments, No. } & 1 & $7(2 \%)$ \\
\hline & 2 & $83(25 \%)$ \\
\hline & 3 & $178(54 \%)$ \\
\hline & 4 and + & $64(19 \%)$ \\
\hline
\end{tabular}

Abbreviations: ECOG, Eastern Cooperative Oncology Group;

${ }^{*}$ ECOG status before beginning therapy with cetuximab $(\mathrm{N}=81)$;

†'One missing data.

Overall, 194 (58\%) cases were positive for the three IHC tests (full-positive cases), while $86(26 \%)$ were full-negative. In the remaining 52 cases (16\%), assessment of EGFR status provided discordant results depending on the kit used. Of the 131 samples negative with the Dako test, 45 (34\%) should be reclassified as positive with at least one of the two others: 29 with the Ventana test and 29 with the Zymed test, including 13 with both. Similarly, $7(3 \%)$ of the samples positive with the Dako test were assessed as negative with at least one of the two other kits ( 5 with Ventana test, 1 with Zymed test, and 1 with both).

Among the 86 full-negative patients, none had EGFR gene amplification in the tumor cells, while all others had balanced EGFR and $C h r 7$ copy numbers.

\subsection{Clinical Outcome and EGFR Status}

Objective response rate, PFS and OS were compared between EGFR-negative or -positive patients by taking into account either results from the Dako test only or results from all IHC tests.

Regarding results from the Dako test only, the ORR did not differ significantly between EGFR-negative and EGFR-positive patients $(P=0.788$; Table 2). In addition, there was no significant difference in terms of PFS (median PFS of 5.2 months CI 95\% [4.0 - 6.4] and 4.1 months CI 95\% [3.0 - 5.2] for EGFR-negative and EGFR-positive patients, respectively; $P=0.326$; Figure 2) or OS (median OS of 14.9 months CI 95\% [12.1 17.7] and 11.4 months CI 95\% [8.1 - 14.6] for EGFR-negative and EGFR-positive patients, respectively; $P=$ 0.888 ; Figure 3 ) between the two populations of patients.

Similarly, comparison of full-negative and other patients (full positive or discordant patients) did not show any significant difference in terms of objective response rate $(P=0.507$; Table 2), PFS (median PFS of 5.9 months CI 95\% [4.4 - 7.5] and 4.0 months CI 95\% [3.0 - 5.1] for full-negative and other patients, respectively; $P$ $=0.222$; Figure 2) or OS (median OS of 12.3 months CI 95\% [8.9 - 15.7] and 13.2 months CI 95\% [10.3 - 16.2] for full-negative and other patients, respectively; $P=0.686$; Figure 3).

\subsection{Safety}

Among the 332 patients for whom safety data were collected, $72(22 \%)$ reported at least one drug-related toxicity of grade III-IV intensity, leading to treatment discontinuation in 17 (24\%) cases.

Regardless of the intensity, the most frequently reported drug-related toxicities were cutaneous, hematologic 
Table 2. Response to treatment according to EGFR status.

\begin{tabular}{|c|c|c|c|c|c|}
\hline \multirow{2}{*}{ EGFR status } & \multicolumn{4}{|c|}{ Objective response } & \multirow{2}{*}{$\begin{array}{c}\text { Chi-2 } \\
\text { P }\end{array}$} \\
\hline & Complete or partial response & Stable disease & Progressive disease & Not evaluable & \\
\hline \multicolumn{6}{|l|}{ Dako test ${ }^{*}$} \\
\hline negative $(\mathrm{N}=131)$ & $29(22 \%)$ & $35(27 \%)$ & $53(40 \%)$ & $14(11 \%)$ & 0.788 \\
\hline positive $(\mathrm{N}=201)$ & $48(24 \%)$ & $44(22 \%)$ & $85(42 \%)$ & $24(12 \%)$ & \\
\hline \multicolumn{6}{|l|}{ All IHC tests ${ }^{\dagger}$} \\
\hline Full-negative cases $(\mathrm{N}=86)$ & $21(24 \%)$ & $24(28 \%)$ & $30(35 \%)$ & $11(13 \%)$ & 0.507 \\
\hline All other cases $(\mathrm{N}=246)$ & $56(23 \%)$ & $55(22 \%)$ & $108(44 \%)$ & $27(11 \%)$ & \\
\hline Total $(\mathrm{N}=332)$ & 77 & 79 & 138 & 38 & \\
\hline
\end{tabular}

Abbreviations: EGFR, epidermal growth factor receptor; IHC, immunohistochemistry; SISH, silver-enhanced in situ hybridization. *EGFR status was defined by considering only the results from the Dako's EGFR pharmDx ${ }^{\mathrm{TM}} \mathrm{kit}$.

'EGFR status was defined by considering the results from all three IHC tests, then confirmed by SISH for full-negative cases.
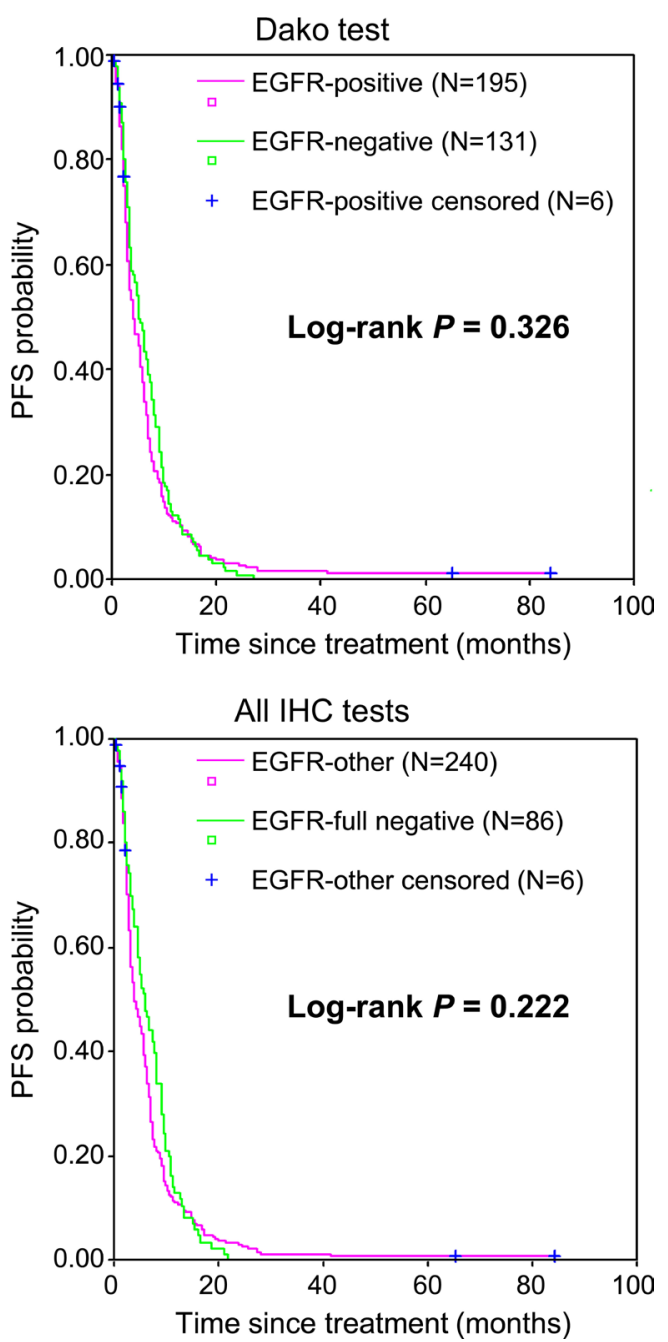

EGFR, epidermal growth factor receptor; IHC, immunohistochemistry; PFS, progression-free survival; SISH, silver-enhanced in situ hybridization.

Figure 2. Progression-free survival according to EGFR status. EGFR status was defined by considering either the results from Dako's EGFR pharmDx ${ }^{\text {TM }}$ kit (Dako test) only (upper panel) or the results from all three IHC tests (all IHC tests), then confirmed by SISH for full-negative cases (lower panel). Data for the 6 patients who did not have the event of interest (progression or death) at the date of the last follow-up visit were censored. 

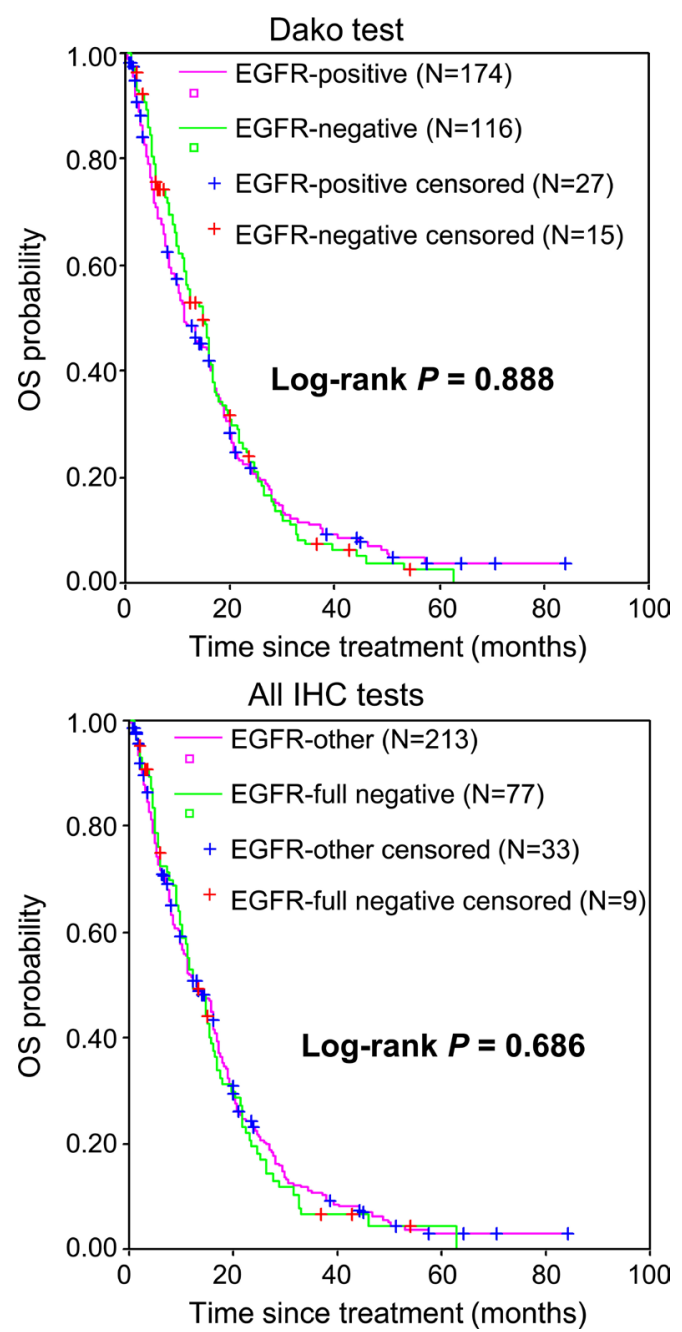

EGFR, epidermal growth factor receptor; IHC, immunohistochemistry; OS, overall survival; SISH, silver-enhanced in situ hybridization.

Figure 3. Overall survival according to EGFR status. EGFR status was defined by considering either the results from Dako's EGFR pharmDx ${ }^{\mathrm{TM}}$ kit (Dako test) only (upper panel) or the results from all three IHC tests (all IHC tests), then confirmed by SISH for full-negative cases (lower panel). Data for the 42 patients still alive or lost to follow-up at the date of the last follow-up visit were censored.

and digestive events, which occurred in $33(10 \%), 15(5 \%)$ and $14(4 \%)$ patients, respectively. No increase of toxicity was associated with EGFR negative or positive status.

\section{Discussions}

"ERBITUX-OUEST" is the first large cohort study that investigated the association between EGFR over expression in IHC using the three commercially available kits with clinical response and survival to cetuximab therapy in mCRC patients who had been treated regardless of their EGFR status. The use of anti-EGFR monoclonal antibodies was per label restricted to EGFR expressing $\mathrm{mCRC}$ to assure that the right drug is given to the right patient. From 2007, the use of KRAS status (exon 2) has become the gold standard [16]-[19]. Although, the EGFR status was and is still subject to discordant results, the use of a biological test is a potential way of achieving it. In this respect, our study clearly indicates that assessment of EGFR status using IHC may provide discordant results depending on the kit used. These discordances may induce false-positive and false-negative results with the consequence of potential misuse of cetuximab in these patients. In addition, we also confirmed, in a large cohort of patients, that IHC EGFR status is not correlated with response rate since mCRC patients with full-negative EGFR status may also benefit from cetuximab, as it was previously suggested in small series 
of patients [6] [8]-[12].

IHC was widely used to screen mCRC patients who are considered for EGFR-targeted therapy until 2008. In an attempt to standardize the assessment of EGFR status, the Dako's EGFR pharmDx ${ }^{\mathrm{TM}}$ kit has received FDA approval for selecting CRC patients eligible for treatment with cetuximab. However, at least two other EGFR detection kits from different commercial sources are currently available (Zymed EGFR kit from Zymed ${ }^{\mathbb{R}}$ Labo- $^{-}$ ratories and Ventana CONFIRM ${ }^{\mathrm{TM}}$ EGFR 3C6 antibody from Ventan ${ }^{\circledR}$ ) and data comparing these antibodies are limited. In current medical practice, clinicians should decide for or against cetuximab treatment based on the results given by their pathologists, regardless of the kit which had been used for EGFR detection. However, our results showed that these tests are clearly not equivalent. EGFR positive expression was detected consistently by the three tests for $58 \%$ of patients with mCRC, i.e., a proportion slightly lower than the $>75 \%$ proportion of EGFR-positive mCRC patients commonly reported in the literature [20] [21]. Actually, 34\% of the Dako-negative cases could be reclassified as positive with one or two of the other tests, suggesting, as previously described [22] [23], that the Dako test is less sensitive than the two others in detecting EGFR expression in mCRC patients. Together our results indicate that EGFR status assessed by IHC did not correlate significantly with ORR, PFS and OS, regardless of whether results from the Dako test only or results from all three IHC tests were taken into account. IHC evaluation of EGFR expression is not a reliable way to screen for mCRC patients who may be suitable for cetuximab therapy and may lead to an underuse of cetuximab.

Having shown that EGFR test kits can lead to cetuximab misuse, our second aim was to describe the potential difference in terms of clinical response between EGFR-negative patients according to the Dako test only, EGFR-discordant patients, full-positive and full-negative patients. Data from "ERBITUX-OUEST" study showed that EGFR-non-detected tumors have the potential to respond to EGFR-therapy, confirming previous findings obtained on smaller series of patients. In accordance with our results, four studies reported $8 \%$ to $30 \%$ response rates in EGFR-non-detected patients treated with either cetuximab and irinotecan or cetuximab alone [6] [8] [10] [12]. In another study, carried out in 9 EGFR-negative patients treated with cetuximab alone, median OS and median PFS were found to be better than those from the pivotal study (BOND study) performed in EGFR-positive patients (10.2 and 7 months versus 8.6 and 4.1 months, respectively) [2] [11]. Over expression of EFGR is usually tested on the primary. No clear data are available on the fact that successive chemotherapy lines could change the status of EGFR in comparison to the one before any treatment. Similarly in the PRIME [16] trial combining FOLFOX and Panitumumab, another anti-EGFR monoclonal antibody, a retrospective analysis of outcome according to EGFR IHC expression using the DAKO kit showed that both EGFR expressing and EGFR non-detected $\mathrm{mCRC}$ had a similar benefit from the addition of Panitumumab to FOLFOX, independent of EGFR expression.

Results from "ERBITUX-OUEST" have also been confirmed in the context of a clinical trial called FOLFIRICETUX. It was a multicenter phase II study conducted in France from October 2005 to January 2008 that included 104 patients with histologically-confirmed mCRC. EGFR status has been retrospectively assessed for 64 patients using the same three available IHC tests. Overall, $13(20 \%)$ cases were negative for the three antibodies and results were conflicting for five (8\%) (i.e., negative with Dako test and positive with at least one of the two others). None of these 18 tumor samples showed EGFR gene amplification as assessed by in situ hybridization. Clinical benefit was observed in seven of the 10 assessable full-negative patients (four partial responses and three stable diseases). Median PFS was 6.6 months for the total population versus 3.8 months for the full-negative patients (difference not statistically significant; $P=0.857$ ). It was thus concluded that full-negative EGFR patients seemed to have the same ORR and the same PFS in comparison to the total population, suggesting that EGFR-negative patients could have the same responses profile and benefit as EGFR-positive patients [24].

Taken together these observations strongly suggest that negative-EGFR staining does not preclude patients from benefiting from EGFR-targeted therapies. Many technical reasons have been advocated for the lack of association between EGFR detected by IHC and response to EGFR-targeted treatment [25]. These reasons include disparity between the form of epitope of EGFR detected by IHC and that targeted by anti-EGFR antibodies, as well as issues related to processing and handling tumor tissue samples, such as prolonged storage [26]. IHC is also a semi-quantitative method that lacks a standardized scoring system and is subject to inter-observer variation. Moreover, differences between primary colorectal tumors and their metastases with regard to EGFR expression have been reported, indicating that reliance on such biomarkers in the primary tumor to predict treatment response of metastatic growths may be inappropriate [27]. 
However, limitations of "ERBITUX-OUEST" study have to be acknowledged, this is an observational study and patients were not randomized with or without Cetuximab, and irinotecan was used in combination. A major strength of the study lies in the fact that the Cancer Observatory population is representative of all the patients treated for mCRC after irinotecan failure in Bretagne and Pays de la Loire areas for a two-year period (20052006) in public and private hospitals. "ERBITUX-OUEST" study thus provides for the first time a "real-world" experience based on the largest set of EGFR-negative patients as yet reported. In addition, a critical point that made our study feasible and so informative is that it was conducted as a multidisciplinary study benefiting from the expertise of pathologists, clinicians and pharmacists.

\section{Conclusions}

Data from "ERBITUX-OUEST" study indicate that pre-therapeutic assessment of EGFR status should be no longer considered for optimizing response to cetuximab based therapy, regardless of the detection kit used (Dako, Zymed or Ventana). The EGFR status is still mentioned in the European label, however, a health institution in France recommends not to research EGFR status since May 2009 [28] and our study tends to give strong argue to change this label. Nevertheless, efforts are still needed to identify response-predicting factors in order to better select patients for EGFR-targeted treatment. Based on data generated after this observational cohort, KRAS [29]-[34] and even more recently K and N-RAS exons 2, 3 and 4 have been shown to be major predictive factors of efficacy [35] [36].

The use of anti-EGFR monoclonal antibodies in the treatment of mCRC provides a way of personalizing treatment based on biomarkers. EGFR expression by IHC does not seem to be reliable, possibly for technical reasons and other predictive biomarkers including RAS mutational status should be preferred.

\section{Acknowledgements}

We would like to thank all clinicians, pharmacists, and directors of public hospitals and private institutions from Bretagne and Pays de la Loire and Jean-Luc Caillot from the Cancéropôle Grand Ouest. Special thanks to Aude de Fallois and Sory Traoré.

\section{Grant Support}

This work was supported by a grant from the Ligue Nationale contre le Cancer (Comité du Finistère) and by a grant from Cancéropole Grand Ouest.

\section{Conflict of Interest}

The following authors have indicated a financial or other interest that is relevant to the subject matter under consideration in this article. Consultant/Advisory Role: Jean-Philippe Metges, Merck Serono, Amgen, Roche; Erick Gamelin, Amgen; Jean-François Ramée, Merck Serono, Amgen; Jean-Luc Raoul, Merck Serono, Amgen; JeanYves Douillard, Merck Serono, Amgen, Roche; Alain Volant, Merck Serono.

\section{References}

[1] Wolpin, B.M. and Mayer, R.J. (2008) Systemic Treatment of Colorectal Cancer. Gastroenterology, 134, $1296-1310$. http://dx.doi.org/10.1053/j.gastro.2008.02.098

[2] Cunningham, D., Humblet, Y., Siena, S., et al. (2004) Cetuximab Monotherapy and Cetuximab plus Irinotecan in Irinotecan-Refractory Metastatic Colorectal Cancer. The New England Journal of Medicine, 351, 337-345. http://dx.doi.org/10.1056/NEJMoa033025

[3] Saltz, L.B., Meropol, N.J., Loehrer Sr., P.J., et al. (2004) Phase II Trial of Cetuximab in Patients with Refractory Colorectal Cancer That Expresses the Epidermal Growth Factor Receptor. Journal of Clinical Oncology, 22, $1201-1208$. http://dx.doi.org/10.1200/JCO.2004.10.182

[4] Mayer, A., Takimoto, M., Fritz, E., et al. (1993) The Prognostic Significance of Proliferating Cell Nuclear Antigen, Epidermal Growth Factor Receptor, and Mdr Gene Expression in Colorectal Cancer. Cancer, 71, 2454-2460. http://dx.doi.org/10.1002/1097-0142(19930415)71:8<2454::AID-CNCR2820710805>3.0.CO;2-2

[5] Nicholson, R.I., Gee, J.M. and Harper, M.E. (2001) EGFR and Cancer Prognosis. European Journal of Cancer, 37, 915. http://dx.doi.org/10.1016/S0959-8049(01)00231-3 
[6] Lenz, H.J., Van Custem, E., Khambata-Ford, S., et al. (2006) Multicenter Phase II and Translational Study of Cetuximab in Metastatic Colorectal Carcinoma Refractory to Irinotecan, Oxaliplatin and Fluoropyrimidines. Journal of Clinical Oncology, 24, 4914-4921.

[7] Italiano, A., Follana, P., Caroli, F.X., et al. (2008) Cetuximab Shows Activity in Colorectal Cancer Patients with Tumors for Which FISH Analysis Does Not Detect an Increase in EGFR Gene Copy Number. Annals of Surgical Oncol$o g y$, 15, 649-654. http://dx.doi.org/10.1245/s10434-007-9667-2

[8] Chung, K.Y., Shia, J., Kemeny, N.E., et al. (2005) Cetuximab Shows Activity in Colorectal Cancer Patients with Tumors That Do Not Express the Epidermal Growth Factor Receptor by Immunohistochemistry. Journal of Clinical Oncology, 23, 1803-1810. http://dx.doi.org/10.1200/JCO.2005.08.037

[9] Saltz, L. (2005) Epidermal Growth Factor Receptor-Negative Colorectal Cancer: Is There Truly Such an Entity? Clinical Colorectal Cancer, 5, 98-100. http://dx.doi.org/10.3816/CCC.2005.s.013

[10] Vincenzi, B., Santini, D. and Tonini, G. (2006) New Issues on Cetuximab Mechanism of Action in Epidermal Growth Factor Receptor-Negative Colorectal Cancer: The Role of Vascular Endothelial Growth Factor. Journal of Clinical Oncology, 24, 1957. http://dx.doi.org/10.1200/JCO.2005.05.0450

[11] Huertas Fernández, M.J., Rodríguez Mateos, M.E., Gómez Reina, M.J., et al. (2007) Treatment with Cetuximab in Metastatic Colorectal Cancer Patients Who Do Not Express the Epidermal Growth Factor Receptor. Farmacia Hospitalaria, 31, 264-269. http://dx.doi.org/10.1016/S1130-6343(07)75389-8

[12] Wierzbicki, R., Jonker, D.J., Moore, M.J., et al. (2011) A Phase II, Multicenter Study of Cetuximab Monotherapy in Patients with Refractory, Metastatic Colorectal Carcinoma with Absent Epidermal Growth Factor Receptor Immunstaining. Investigational New Drugs, 29, 167-174.

[13] Francoual, M., Etienne-Grimaldi, M.C., Formento, J.L., et al. (2006) EGFR in Colorectal Cancer: More than a Simple Receptor. Annals of Oncology, 17, 962-967. http://dx.doi.org/10.1093/annonc/mdl037

[14] Hofmann, M., Stoss, O., Shi, D., et al. (2008) Assessment of a HER2 Scoring System for Gastric Cancer: Results from a Validation Study. Histopathology, 52, 797-805. http://dx.doi.org/10.1111/j.1365-2559.2008.03028.x

[15] Rüschoff, J., Dietel, M., Baretton, G., et al. (2010) HER2 Diagnostics in Gastric Cancer-Guideline Validation and Development of Standardized Immunohistochemical Testing. Virchows Arch, 457, 299-307. http://dx.doi.org/10.1007/s00428-010-0952-2

[16] Douillard, J.Y., Siena, S., Cassidy, J., et al. (2010) Randomized, Phase III Study of Panitumumab with Infusional Fluorouracil, Leucovorin, and Oxaliplatin (FOLFOX4) versus FOLFOX4 Alone as First-Line Treatment in Patients with Previously Untreated Metastatic Colorectal Cancer: The PRIME Study. Journal of Clinical Oncology, 28, 4697- 4705.

[17] Van Cutsem, E., Peeters, M., Siena, S., Humblet, Y., Hendlisz, A., Neyns, B., Canon, J.L., Van Laethem, J.L., Maurel, J., Richardson, G., Wolf, M. and Amado, R.G. (2007) Open-Label Phase III Trial of Panitumumab plus Best Supportive Care Compared with Best Supportive Care Alone in Patients with Chemotherapy-Refractory Metastatic Colorectal Cancer. Journal of Clinical Oncology, 25, 1658-1664. http://dx.doi.org/10.1200/JCO.2006.08.1620

[18] Amado, R.G., Wolf, M., Peeters, M., Van Cutsem, E., Siena, S., Freeman, D.J., Juan, T., Sikorski, R., Suggs, S., Radinsky, R., Patterson, S.D. and Chang, D.D. (2008) Wild-Type KRAS Is Required for Panitumumab Efficacy in Patients with Metastatic Colorectal Cancer. Journal of Clinical Oncology, 26, 1626-1634. http://dx.doi.org/10.1200/JCO.2007.14.7116

[19] Bokemeyer, C., Van Cutsem, E., Rougier, P., et al. (2012) Addition of Cetuximab to Chemotherapy as First-Line Treatment for KRAS Wild-Type Metastatic Colorectal Cancer: Pooled Analysis of CRYSTAL and OPUS Randomized Clinical Trials. European Journal of Cancer, 48, 1466-1475.

[20] Adenis, A., Aranda, A.E., Robin, Y.M., et al. (2005) Expression of the Epidermal Growth Factor Receptor (EGFR or HER1) and Human Epidermal Growth Factor Receptor 2 (HER2) in a Large Scale of Metastatic Colorectal Cancer (mCRC) Trial. Journal of Clinical Oncology, 23, 3630.

[21] Goldstein, N.S. and Armin, M. (2001) Epidermal Growth Factor Receptor Immunohistochemical Reactivity in Patients with American Joint Committee on Cancer Stage IV Colon Adenocarcinoma: Implications for a Standardized Scoring System. Cancer, 92, 1331-1346. http://dx.doi.org/10.1002/1097-0142(20010901)92:5<1331::AID-CNCR1455>3.0.CO;2-M

[22] Penault-Llorca, F., Cayre, A., Arnould, L., et al. (2006) Is There an Immunohistochemical Technique Definitively Valid in Epidermal Growth Factor Receptor Assessment? Oncology Reports, 16, 1173-1179. http://dx.doi.org/10.3892/or.16.6.1173

[23] Buckley, A.F. and Kakar, S. (2007) Comparison of the Dako EGFR pharmDx Kit and Zymed EGFR Antibody for Assessment of EGFR Status in Colorectal Adenocarcinoma. Applied Immunohistochemistry \& Molecular Morphology, 15, 305-309. http://dx.doi.org/10.1097/01.pai.0000213141.47277.bf

[24] Metges, J.P., Boisdron-Celle, M., Morel, A., et al. (2010) Full Negative EGFR Status by Immunochemistry and FISH 
and Response to Personalized Treatment Using Cetuximab and FOLFIRI. 7th Gastrointestinal Cancers Symposium of the American Society of Clinical Oncology, Orlando, 22-24 January 2010.

[25] Shia, J., Klimstra, D.S., Li, A.R., et al. (2005) Epidermal Growth Factor Receptor Expression and Gene Amplification in Colorectal Carcinoma: An Immunohistochemical and Chromogenic in Situ Hybridization Study. Modern Pathology, 18, 1350-1356. http://dx.doi.org/10.1038/modpathol.3800417

[26] Atkins, D., Reiffen, K.A., Tegtmeier, C.L., Winther, H., Bonato, M.S. and Störkel, S. (2004) Immunohistochemical Detection of EGFR in Paraffin-Embedded Tumor Tissues: Variation in Staining Intensity Due to Choice of Fixative and Storage Time of Tissue Sections. Journal of Histochemistry \& Cytochemistry, 52, 893-901. http://dx.doi.org/10.1369/jhc.3A6195.2004

[27] Scartozzi, M., Bearzi, I., Berardi, R., et al. (2004) Epidermal Growth Factor Receptor (EGFR) Status in Primary Colorectal Tumors Does Not Correlate with EGFR Expression in Related Metastatic Sites: Implications for Treatment with EGFR-Targeted Monoclonal Antibodies. Journal of Clinical Oncology, 22, 4772-4778. http://dx.doi.org/10.1200/JCO.2004.00.117

[28] French National Authority for Health (2009) Erbitux, Opinion of the Commission of Transparency. http://www.has-sante.fr/portail/upload/docs/application/pdf/2009-09/erbitux - ct-6366.pdf

[29] Lièvre, A., Bachet, J.B., Le Corre, D., et al. (2006) KRAS Mutation Status Is Predictive of Response to Cetuximab Therapy in Colorectal Cancer. Cancer Research, 66, 3992-3995. http://dx.doi.org/10.1158/0008-5472.CAN-06-0191

[30] Di Fiore, F., Blanchard, F., Charbonnier, F., et al. (2007) Clinical Relevance of KRAS Mutation Detection in Metastatic Colorectal Cancer by Cetuximab plus Chemotherapy. British Journal of Cancer, 96, 1166-1169. http://dx.doi.org/10.1038/sj.bjc.6603685

[31] Khambata-Ford, S., Garrett, C.R., Meropol, N.J., et al. (2007) Expression of Epiregulin and Amphiregulin and K-ras Mutation Status Predict Disease Control in Metastatic Colorectal Cancer Patients Treated with Cetuximab. Journal of Clinical Oncology, 25, 3230-3237. http://dx.doi.org/10.1200/JCO.2006.10.5437

[32] Lièvre, A., Bachet, J.B., Boige, V., et al. (2008) KRAS Mutations as an Independent Prognostic Factor in Patients with Advanced Colorectal Cancer Treated with Cetuximab. Journal of Clinical Oncology, 26, 374-379. http://dx.doi.org/10.1200/JCO.2007.12.5906

[33] De Roock, W., Piessevaux, H., De Schutter, J., et al. (2008) KRAS Wild-Type State Predicts Survival and Is Associated to Early Radiological Response in Metastatic Colorectal Cancer Treated with Cetuximab. Annals of Oncology, 19, 508515. http://dx.doi.org/10.1093/annonc/mdm496

[34] De Roock, W., Jonker, D.J., Di Nicolantonio, F., et al. (2010) Association of KRAS p.G13D Mutation with Outcome in Patients with Chemotherapy-Refractory Metastatic Colorectal Cancer Treated with Cetuximab. JAMA: The Journal of the American Medical Association, 304, 1812-1820. http://dx.doi.org/10.1001/jama.2010.1535

[35] Douillard, J.Y., Oliner, K.S., Siena, S., et al. (2013) Panitumumab-FOLFOX4 Treatment and RAS Mutations in Colorectal Cancer. The New England Journal of Medicine, 369, 1023-1034. http://dx.doi.org/10.1056/NEJMoa1305275

[36] Heinemann, V., von Weikersthal, L.F., Decker, T., et al. (2014) FOLFIRI plus Cetuximab versus FOLFIRI plus Bevacizumab as First-Line Treatment for Patients with Metastatic Colorectal Cancer (FIRE-3): A Randomised, OpenLabel, Phase 3 Trial. The Lancet Oncology, 15, 1065-1075. 


\section{Submit or recommend next manuscript to SCIRP and we will provide best service for you:}

Accepting pre-submission inquiries through Email, Facebook, Linkedin, Twitter, etc A wide selection of journals (inclusive of 9 subjects, more than 200 journals)

Providing a 24-hour high-quality service

User-friendly online submission system

Fair and swift peer-review system

Efficient typesetting and proofreading procedure

Display of the result of downloads and visits, as well as the number of cited articles

Maximum dissemination of your research work

Submit your manuscript at: http://papersubmission.scirp.org/ 